\section{De novo DNA methylation by Dnmt3a and Dnmt3b is dispensable for nuclear reprogramming of somatic cells to a pluripotent state}

\author{
Mathias Pawlak $^{1}$ and Rudolf Jaenisch ${ }^{1,2,3}$ \\ ${ }^{1}$ The Whitehead Institute for Biomedical Research, Cambridge, \\ Massachusetts 02142, USA; ${ }^{2}$ Department of Biology, \\ Massachusetts Institute of Technology, Cambridge, \\ Massachusetts 02139, USA
}

Induced pluripotent stem cells (iPSCs) are generated from somatic cells by the transduction of defined transcription factors, and this process involves dynamic changes in DNA methylation. While the reprogramming of somatic cells is accompanied by demethylation of pluripotency genes, the functional importance of de novo DNA methylation has not been clarified. Here, using loss-of-function studies, we generated iPSCs from fibroblasts that were deficient in de novo DNA methylation mediated by Dnmt3a and Dnmt3b. These iPSCs reactivated pluripotency genes, underwent self-renewal, and showed restricted developmental potential that was rescued upon reintroduction of Dnmt3a and Dnmt3b. We conclude that de novo DNA methylation by Dnmt3a and Dnmt3b is dispensable for nuclear reprogramming of somatic cells.

Supplemental material is available for this article.

Received July 19, 2010; revised version accepted March 18, 2011.

Pluripotent stem cells can be generated from somatic cells by viral transduction of the four transcription factors Oct4, Sox2, Klf4, and c-Myc. These induced pluripotent stem cells (iPSCs) are similar to embryonic stem (ES) cells expressing pluripotency genes such as Nanog and Oct4, and are capable of differentiation into cells of all three germ layers in teratoma assays. Furthermore, mouse iPSCs contribute to the germline in chimeric mice and "all iPSC" mice can be derived through tetraploid complementation (Takahashi and Yamanaka 2006; Maherali et al. 2007; Okita et al. 2007; Wernig et al. 2007; Boland et al. 2009; Kang et al. 2009; Zhao et al. 2009). iPSCs generated from human sources are thought to be useful for drug discovery and regenerative medicine (Yamanaka 2009). In fact, in two proof-of-principle studies, iPSCs have been shown to alleviate symptoms in mouse models of sickle cell anemia and Parkinson's disease (Hanna et al. 2007; Wernig et al. 2008).

[Keywords: nuclear reprogramming; iPSCs; de novo DNA methylation; Dnmt3a; Dnmt3b]

${ }^{3}$ Corresponding author.

E-MAIL jaenisch@wi.mit.edu; FAX (617) 258-6505.

Article is online at http://www.genesdev.org/cgi/doi/10.1101/gad.2039011.
The molecular events leading from a differentiated to a pluripotent cell during iPSC derivation are only emerging and remain largely unknown (Hochedlinger and Plath 2009). Studies have shown that distinct markers are activated or repressed sequentially after the induction of reprogramming, with the repression of differentiation genes occurring early and the activation of genes essential for pluripotency, such as Oct4 or Nanog, occurring late during this process (Brambrink et al. 2008; Stadtfeld et al. 2008). The epigenetic state of the differentiated donor cell is completely reprogrammed, and DNA methylation and histone marks of the iPSC resemble that of ES cells (Maherali et al. 2007; Wernig et al. 2007; Mikkelsen et al. 2008).

Changes in DNA methylation patterns are essential for successful nuclear reprogramming, exemplified by the necessity for loss of promoter methylation in pluripotency genes in iPSCs (Takahashi and Yamanaka 2006). If loss of DNA methylation is not achieved, the cells will be only partially reprogrammed (Mikkelsen et al. 2008). Several studies have investigated de novo DNA methylation during the reprogramming process. For example, it has been documented that de novo DNA methyltransferases are highly induced upon reprogramming, and it is known that mouse ES cells have a very high expression of these epigenetic regulators (Okano et al. 1998; Stadtfeld et al. 2008). Also, two studies found increased DNA methylation in certain genomic regions in iPSCs, with the functional importance of this finding remaining elusive (Deng et al. 2009; Doi et al. 2009). However, it has not been resolved whether de novo DNA methylation is important or might even be essential for nuclear reprogramming of somatic cells to a pluripotent state.

De novo DNA methylation in mammals is mediated by Dnmt3a and Dnmt3b (Li 2002) and knockout studies in mice have shown that de novo DNA methylation is essential for development, since Dnmt3a-deficient animals die several weeks after birth and Dnmt3b-deficient animals die in utero (Okano et al. 1999). Further experimentation has shown that fibroblasts lacking Dnmt3b are prone to spontaneous immortalization and genomic instability (Dodge et al. 2005), although mouse ES cells can tolerate the complete absence of DNA methylation (Meissner et al. 2005). DNA methylation by Dnmt3a has a crucial role for imprinting in development (Kaneda et al. 2004).

In this study, we sought to investigate the role of Dnmt3a and Dnmt3b in the generation of iPSCs. Using mouse embryonic fibroblasts (MEFs) in which Dnmt3a and Dnmt3b were conditionally deleted through Cre recombinase, we show that nuclear reprogramming is achieved in the absence of the de novo DNA methyltransferases Dnmt3a and Dnmt3b.

\section{Results and Discussion \\ Generation of iPSCs in the absence of the de novo DNA methyltransferases Dnmt3a and Dnmt3b}

To ask whether somatic cells can be reprogrammed into a pluripotent cell in the absence of de novo DNA methylation, we used MEFs that were homozygous for floxed alleles of both Dnmt3a and Dnmt3b. Two 
complementary approaches were used to generate iPSCs (Fig. 1A). In the first approach, we transduced doubleconditional MEFs (referred to as 3ab MEFs) with lentiviruses encoding the reprogramming factors Oct4, Sox2, c-Myc, and Klf4 and the reverse tetracycline transactivator M2rtTA (Brambrink et al. 2008; Hockemeyer et al. 2008). After transduction, the cells were infected with an adenovirus containing a Cre recombinase and GFP. FACS (fluorescence-activated cell sorting) was used to isolate GFP-positive cells, and reprogramming was initiated 1 d later by adding doxycycline (DOX) to the medium. Since it is known that Dnmt3a and Dnmt3b are expressed at low levels in somatic cells /Okano et al. 1998), we aimed to exclude the possibility of remaining functional Dnmt3a and Dnmt3b after the initiation of reprogramming. To this end, in the second approach, $3 \mathrm{ab}$ MEFs were first infected with the adenovirus harboring the Cre recombinase, passaged several times, and then transduced with the individual reprogramming factors. Similarly to wild-type MEFs, addition of DOX to $3 \mathrm{ab}$ MEFs induced morphological changes within several days in both approaches (Supplemental Fig. S1A). After 17-28 $\mathrm{d}$ of DOX treatment, single colonies morphologically resembling ES cells were subcloned. To select for DOXindependent clones, DOX was withdrawn from the medium shortly after subcloning.

To characterize 3ab-deficient iPSCs, immunostaining was performed. 3ab-deficient iPSCs were found to be positive for the stem cell markers alkaline phosphatase, SSEA-1, Oct4, and Nanog (Fig. 1B; Supplemental Fig. S1B$\mathrm{D})$, and the staining patterns were indistinguishable from those of control V6.5 ES cells. The expression of the pluripotency gene Nanog was also investigated by quan-

A

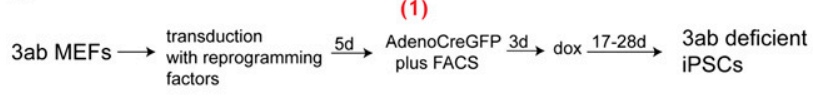

(2)

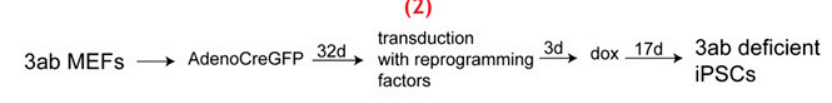

B

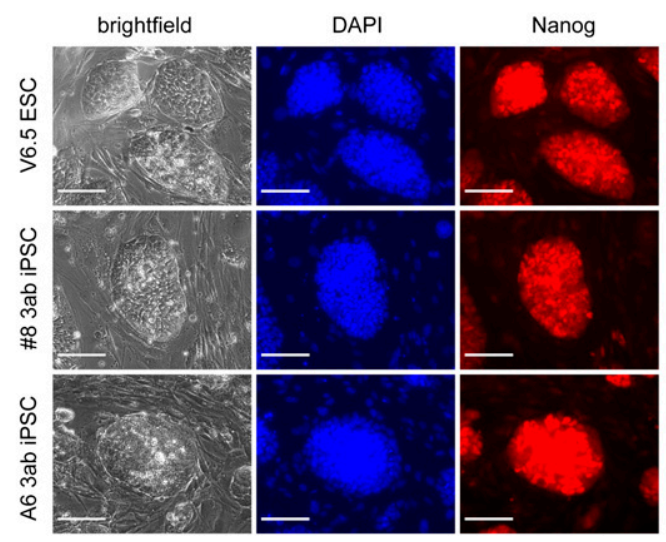

Figure 1. Generation of iPSCs in the absence of de novo DNA methyltransferase activity. (A) Schematic of the two approaches to reprogram conditional knockout MEFs for Dnmt3a and Dnmt3b. MEFs were infected with adenovirus harboring a Cre recombinase and GFP either after or before infection with inducible lentiviruses encoding the reprogramming factors Oct4, Sox2, c-Myc and Klf4. (B) Immunostaining for the pluripotency marker Nanog. Bars, $100 \mu \mathrm{m}$. titative RT-PCR (qRT-PCR) and was found to be expressed in 3ab-deficient iPSCs at levels comparable with ES cells (Supplemental Fig. S1E). These results suggest that reprogramming can occur in the absence of de novo DNA methylation through Dnmt3a and Dnmt3b.

Upon differentiation of ES cells, pluripotency genes such as Oct4 and Nanog are highly methylated, reflecting stable long-term silencing (Feldman et al. 2006), while iPSC generation leads to demethylation, consistent with reactivation of Oct4 and Nanog. As expected, bisulfite sequencing demonstrated that the Nanog gene became demethylated in 3ab-deficient iPSCs, comparable with V6.5 ES cells (Supplemental Fig. S1F).

To evaluate whether the Dnmt3a and Dnmt3b alleles had been deleted by Cre recombinase, Southern blot analyses were performed. Individual colonies were subcloned from iPSCs derived from FACS-isolated AdenoCre-GFP-infected MEFs. Figure 2A shows that all Dnmt3a and Dnmt3b alleles were deleted, confirming that iPSCs can be derived in the absence of de novo DNA methylation. Similarly, $>20$ subclones generated with the second approach had deleted all Dnmt3a and Dnmt3b alleles (Fig. 2B).

To confirm the independent origin of 3ab-deficient iPSC lines, we performed Southern blot analysis with a probe that recognizes both endogenous and transgenic Oct4. The majority of the 18 iPSC lines randomly chosen for this analysis had a unique integration pattern and thus were of independent origin (Supplemental Fig. S2A). The number of integrations for the Oct4 provirus varied between two and eight, with clones \#8 and B2 having the lowest and clone A6 having the highest number of integrations. Expression analysis of Oct4 proviral transcription correlated with basal vector expression, with clone A6 showing some residual transgene expression (Supplemental Fig. S2B). Also, we observed different numbers of proviral integrations for the other reprogramming factors, with clone B2 lacking a c-Myc proviral integration (Supplemental Fig. S3).

It is known from nuclear transfer studies that hypomethylation of somatic donor cells increases reprogramming efficiency (Blelloch et al. 2006). To ask whether reprogramming was enhanced in the absence of de novo DNA methylation, we infected $3 \mathrm{ab}$ conditional MEFs with either an Adeno-GFP or an Adeno-Cre-GFP virus and sorted equal amounts of GFP-positive cells. Then, we induced reprogramming by transduction with a polycistronic vector harboring the four reprogramming factors (Sommer et al. 2009). After $16 \mathrm{~d}$ of reprogramming, we omitted DOX and counted colonies on day 19. We observed only a slight difference in colony number between control and 3ab-deficient samples (1.5-fold), suggesting that there is no major effect on reprogramming in the absence of de novo DNA methylation. One representative plate was stained for alkaline phosphatase, a commonly used pluripotency marker (Supplemental Fig. S4).

\section{Molecular characterization of 3ab-deficient iPSCs}

It was shown previously that a global change in DNA methylation patterns occurs during iPSC derivation (Mikkelsen et al. 2008). We therefore investigated the methylation of genomic repeat sequences during reprogramming in the absence of de novo DNA methylation. To this end, we compared 3ab-deficient with control iPSCs or V6.5 ES cells. Southern blot analysis with the 
A

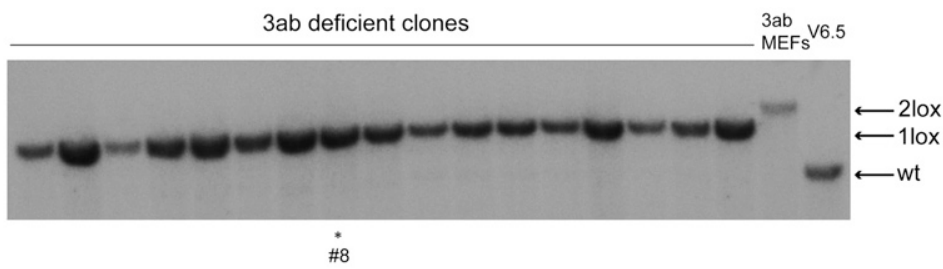

Dnmt3b

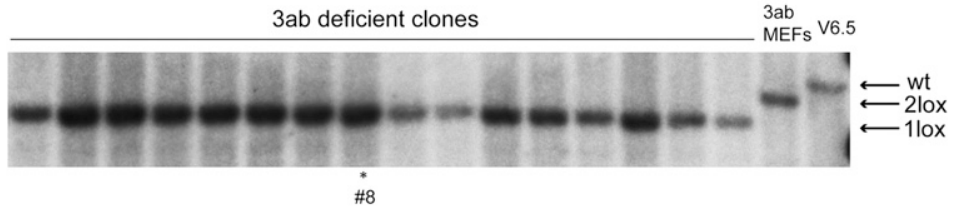

B
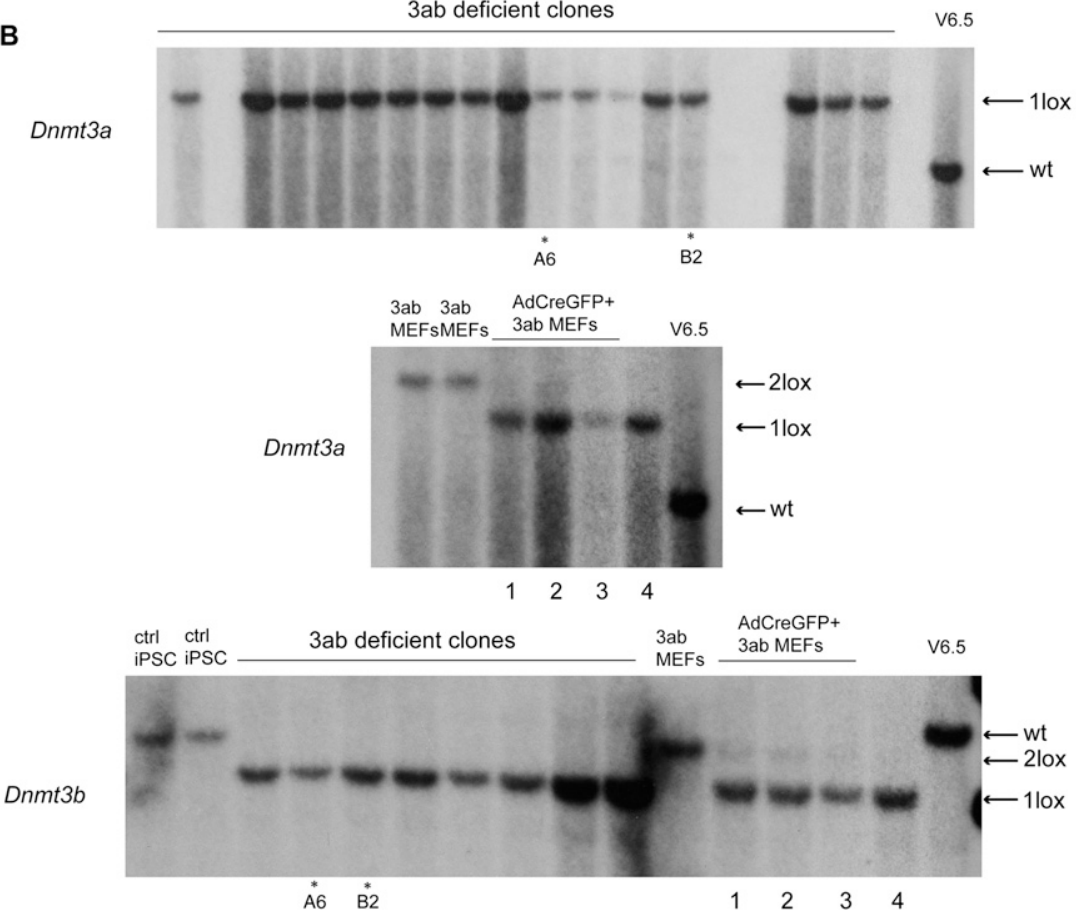

Figure 2. Characterization of 3ab-deficient iPSC lines. (A) Southern blot analysis shows $3 \mathrm{ab}$-deficient iPSC lines obtained through approach 1 (Fig. 1A). Clone \#8 is indicated by an asterisk. $(B)$ Southern blot analysis of 3ab-deficient iPSC lines obtained through approach 2 (Fig. 1A). Clones A6 and B2 are indicated by an asterisk. Additionally, 3ab MEFs infected with Adeno-Cre-GFP are shown before the transduction with the reprogramming factors and over the course of several passages (1-3). Lane 4 displays DNA from a culture dish containing mixed iPSC colonies. Note that the loop-out induced by Cre recombinase was highly efficient. "2lox" represents the conditional alleles and "1lox" represents the deficient alleles of Dnmt3a and Dnmt3b, respectively.

methylation-sensitive enzyme HpaII showed that 3abdeficient iPSC lines had slightly decreased DNA methylation levels of minor satellite repeats and intracisternal $\mathrm{A}$ particles (IAPs) (Supplemental Fig. S5), consistent with previous results showing reduced IAP methylation in Dnmt3a and Dnmt3b mutant cells (Okano et al. 1999). Since it has been demonstrated that female ES cells are hypomethylated (Zvetkova et al. 2005), we determined the gender of the 3ab-deficient iPSC lines and found that male clone \#8 and female clones A6 and B2 had similar methylation levels of the genomic repeat sequences.

To investigate global gene expression in 3ab-deficient iPSCs, we performed expression profiling (Supplemental
Fig. S6). This analysis revealed that 165 genes were $>2.5$-fold up-regulated in 3abdeficient iPSCs and 116 genes were 2.5fold down-regulated in comparison with control iPSC lines (Supplemental Table S1). A large proportion of the up-regulated genes were X-linked, such as genes of the rhox cluster, a known target for Dnmt3a and Dnmt3b. In fact, loss of Dnmt3a and Dnmt3b in ES cells leads to activation of the rhox cluster, which is normally silenced (Oda et al. 2006). Furthermore, it was shown that ES cells that lack both de novo and maintenance DNA methyltransferases up-regulate genes on the $\mathrm{X}$ chromosome (Fouse et al. 2008). We found Gt12 and Rian, two members of the Dlk1-Dio3 cluster, up-regulated in 3abdeficient iPSCs, indicating that Dnmt3a/ b-mediated DNA methylation may be involved in the regulation of this genomic region, as suggested previously (Kato et al. 2007). For functional annotation, we performed gene ontology (GO) analyses of the differentially regulated genes in 3abdeficient iPSCs (Supplemental Table S2). Top GO terms for the up-regulated genes were chromosome organization (GO: 0051276) and organelle organization (GO: 0006996). However, more specialized categories such as meiosis (GO: 0007126) or reproductive cellular process (GO: 0048610) were also observed, and it is known that Dnmt3a plays an important role in germ cell development (Kaneda et al. 2004; Kato et al. 2007). The top GO terms for the down-regulated genes were in response to stimulus (GO: 0050896) and multicellular organismal development (GO: 0007275), with other more specialized developmental GO terms. It was noteworthy that up-regulated genes would rather be grouped into the intracellular region (GO: 0005622), whereas down-regulated genes would fall into the extracellular region (GO: 0005576). In summary, our data suggest that 3ab-deficient iPSCs display an expression profile highly similar to control iPSCs, yet with some noticeable differences.

A previous study grouped gene promoters in mouse ES cells into either methylated or unmethylated (Fouse et al. 2008). We compared our gene list of differentially expressed genes in 3ab-deficient iPSCs with the gene list of methylated and unmethylated promoters to evaluate whether we could identify a correlation between the lack of de novo DNA methylation and the observed expression profile. We found that 59 of the differentially expressed genes in 3ab-deficient iPSCs are methylated and 55 genes are unmethylated in mouse ES cells (Supplemental Table S3). Using a different data set (Meissner et al. 2008), we did not observe a preferred distribution of the differentially regulated genes into methylated or unmethylated in normal mouse ES cells in comparison with MEFs 
(Supplemental Fig. S7). Additionally, sites of enhanced DNA methylation in human iPSCs in relation to human ES cells were reported previously (Doi et al. 2009). However, differentially expressed genes in 3ab-deficient iPSCs did not significantly overlap with these sites. These data suggest that, while expression of some genes in ES cells may be affected by the state of DNA methylation, this effect may not be direct.

\section{Developmental potential of Dnmt3a-and Dnmt3b-deficient iPSCs}

To assess developmental potential, 3ab-deficient iPSCs were injected into the flanks of SCID /severe combined immunodeficient) mice. The resulting teratomas showed mostly ectodermal structures and few endodermal-like, but no well-differentiated, mesodermal structures (Fig. $3 \mathrm{~A})$, suggesting that $3 \mathrm{ab}$-deficient iPSCs have only a restricted developmental potential. Furthermore, the overall size of teratomas derived from 3ab-deficient iPSCs was considerably smaller than that of teratomas from control V6.5 ES cells (data not shown) or control $3 \mathrm{ab}^{+}$iPSCs that were able to contribute to all three germ layers (Supplemental Fig. S8). This is in agreement with previous studies demonstrating that high-passage Dnmt $3 a$ - and $D n m t 3 b$-deficient ES cells were unable to form teratomas, likely because of a decreased level of global DNA methylation. Consistent with this conclusion, developmental potential in Dnmt3a/3b-deficient ES cells could be restored by reintroduction of $D n m t 3 a$ and $D n m t 3 b$ and reestablishment of DNA methylation patterns (Chen et al. 2003).

To investigate whether developmental potential could be restored to $3 \mathrm{ab}$-deficient iPSCs, we reintroduced functional Dnmt3a and Dnmt3b by lentivirus-mediated gene transfer (Supplemental Fig. S9). Indeed, the rescued lines formed teratomas that displayed differentiated cells from all three germ layers (Fig. 3A). This observation suggested that $3 \mathrm{ab}$-deficient iPSCs had been reprogrammed yet could not differentiate, merely due to the
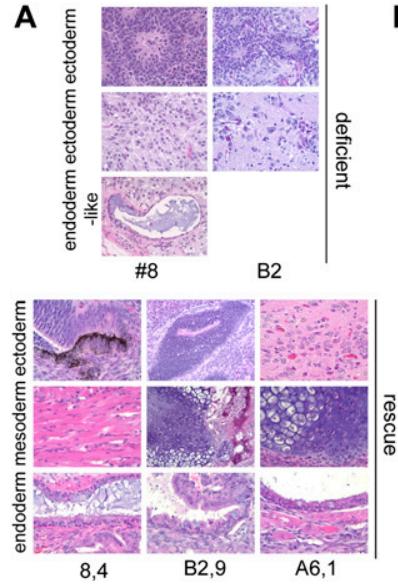

B

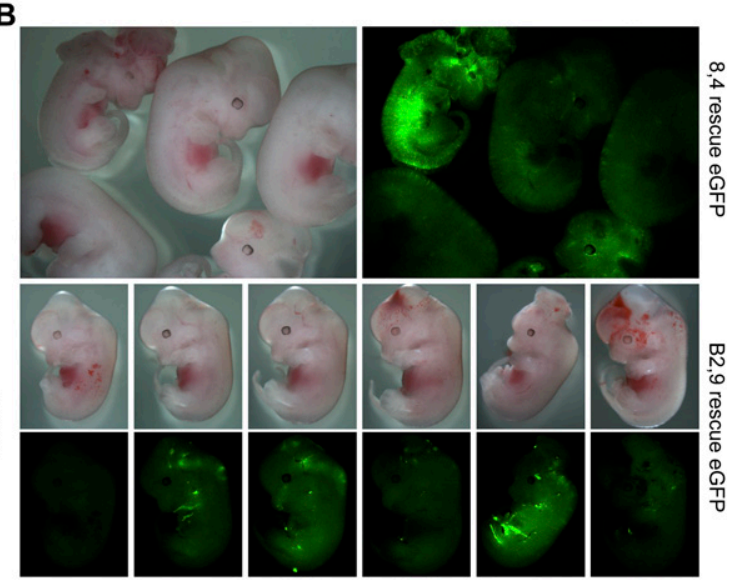

lack of Dnmt3a and Dnmt3b that were rescued upon their reintroduction. As a more stringent assay for pluripotency, we injected eGFP-marked 3ab-deficient iPSCs and rescued lines into blastocysts and harvested the resulting embryos at mid-gestation (Supplemental Fig. S9). We were able to obtain chimeric embryos with clearly visible eGFP signals, indicative of a contribution from the rescued lines, underscoring their developmental potential (Fig. 3B). The degree of chimerism varied between individual embryos. 3ab-deficient iPSCs did not contribute to developing embryos based on the lack of an eGFP signal (data not shown), which was confirmed by the absence of immunohistochemical staining for GFP (Supplemental Fig. S10). In some high-contribution chimeras from the rescued lines, developmental malformations were observed (Fig. 3B) that are likely due to constitutive expression of the lentivirus-transduced Dnmt3a and Dnmt3b genes. In summary, reintroduction of Dnmt3a and Dnmt3b restored pluripotency to the Dnmt3a- and Dnmt3b-deficient iPSCs.

\section{Conclusions}

In this study, we used conditional knockout MEFs to generate iPSCs in the absence of de novo DNA methylation through Dnmt3a and Dnmt3b. We observed robust reactivation of pluripotency markers in $3 \mathrm{ab}$-deficient iPSCs, and their derivation appeared to be similarly efficient compared with wild-type cells. The methylation status of genomic repeat sequences in $3 \mathrm{ab}$-deficient iPSCs was slightly decreased. The global expression profile of 3ab-deficient iPSCs closely resembled that of control iPSCs, although the expression of a small number of genes was changed in 3ab-deficient iPSCs. The question of how gene silencing is achieved in 3ab-deficient iPSCs remains a particularly interesting one. A recent study suggested that gene silencing in mouse ES cells can be achieved by alternative mechanisms to DNA methylation, such as histone methylation by ESET (also known as KMT1E) (Matsui et al. 2010). Whether such mechanisms assure gene silencing in 3ab-deficient iPSCs deserves further study. 3ab-deficient iPSCs formed teratomas that were smaller than teratomas from control lines and had a restricted developmental potential. However, developmental potential could be rescued upon reintroduction of Dnmt3a and Dnmt3b into 3ab-deficient iPSCs, consistent with de novo DNA methylation mediated by Dnmt3a and Dnmt3b being crucial for normal development. In summary, our results provide evidence that the genome of a differentiated cell that is deficient for this major epigenetic regulatory system can be reprogrammed to a pluripotent state.

\section{Materials and methods}

Figure 3. 3ab-deficient iPSCs form teratomas with a restricted developmental potential. $(A)$ Teratomas from \#8 and B2 3ab-deficient iPSCs. Reintroduction of functional Dnmt3a and Dnmt3b into $3 \mathrm{ab}$-deficient iPSCs rescues the ability to efficiently form cells from the three germ layers in a teratoma assay. $(B)$ eGFP-marked rescue lines were injected into blastocysts, and resulting chimeric embryos at mid-gestation are shown. The eGFP signal was clearly visible in the majority of embryos, whereas the degree of chimerism varied. Two embryos with developmental defects are shown. The first embryo of the bottom panel did not show any eGFP signal and serves as a reference of background.

\section{Cell culture and iPSC derivation}

MEFs were isolated from double-homozygous mice for the conditional alleles of Dnmt3a and iPSCs were generated with inducible lentiviruses Dnmt3b (Lin et al. 2006; Nguyen et al. 2007). 
as reported previously (Brambrink et al. 2008; Hockemeyer et al. 2008). Recombination of the conditional alleles of Dnmt3a and Dnmt $3 b$ was induced by infection with adenovirus harboring Cre recombinase and GFP (Ad5CMVCre-eGFP, Gene Transfer Vector Core, University of Iowa). Control iPSC lines were derived from 3ab MEFs without infection with Adeno-Cre-GFP. However, for the efficiency experiment using a previously described DOX-inducible polycistronic vector (Sommer et al. 2009), we included an adenovirus containing only GFP (Ad5CMV-eGFP) to generate the control sample.

\section{Generation of rescue lines and targeting of the Rosa26 locus.}

Full-length Dnmt3a and Dnmt3b were introduced with lentiviral vectors into 3ab-deficient iPSC lines. Both 3ab-deficient and rescue lines were targeted in the Rosa26 locus with eGFP for the purpose of blastocyst injections.

\section{Western blot analysis}

The antibodies to detect Dnmt3a and Dnmt3b were purchased from Imgenex (IMG-268A and IMG-184A).

\section{Southern blot analysis}

Southern blot analyses were used to investigate the loop-out status of the Dnmt3a and Dnmt3b conditional alleles using previously described probes (Lin et al. 2006; Nguyen et al. 2007). As probes for proviral integration analyses, fragments of the cDNAs for the reprogramming factors were excised from the respective lentiviral vectors. The probes for minor satellite repeats (pMR150) and IAPs were described previously (Chapman et al. 1984; Walsh et al. 1998).

\section{Immunostaining and immunohistochemistry}

Immunostaining was described previously (Wernig et al. 2007). Antibodies used were for Oct4 (C10, Santa Cruz Biotechnology), SSEA-1 (Developmental Studies Hybridoma Bank), and Nanog (A300-397A, Bethyl Laboratories). Alkaline phosphatase staining was performed with the Vector Red substrate kit (Vector Laboratories) according to the manufacturer's instructions. The antibody against GFP was ab290 (Abcam). Images for the immunostainings were taken with a Nikon Eclipse Ti microscope and processed equally between samples with Photoshop.

\section{Bisulfite sequencing}

Two micrograms of phenol-chloroform-extracted genomic DNA was converted with bisulfite using the Epitect kit from Qiagen. The genespecific PCR primers recognizing bisulfite-treated DNA were described previously for Nanog (Imamura et al. 2006). PCR-amplified products were gel-purified and cloned into TOPO pCR2.1 from Invitrogen and sequenced with M13 primers.

\section{qRT-PCR analysis}

Primers for Nanog qRT-PCR analysis were described previously (Stadtfeld et al. 2008). Primers used to detect total Oct4 were described elsewhere (Hanna et al. 2009). Primers to detect transgenic Oct4 were GCTCAGT GATGCTGTTGATCAGG (forward) and CAAAGGCATTAAAGCAGCG TATC (reverse).

\section{Expression profiling}

Total RNA was isolated with the RNeasy kit from Qiagen. RNA was labeled with the QuickAmp kit from Agilent and hybridized to $4 \times 44 \mathrm{k}$ mouse whole-genome arrays from Agilent. The two-color microarrays were normalized across channels by lowess (locally weighted scatter plot smoothing) and between arrays by quantile normalization of average intensities (Aquantile), as implemented by the limma package in Bioconductor. Differential expression was defined by an expression ratio of at least 2.5 -fold. To reduce any potential gender bias, expression ratios were calculated giving equal weight to male (\#8) and female (mean of A6 and B2) 3ab-deficient iPSC lines and to male (mean of \#3, \#4, and \#5) and female (\#6) control iPSC lines. The scatter plot (Supplemental Fig. S6) highlights probes that are at least 2.5-fold differentially expressed in 3ab-deficient iPSC lines compared with controls, as listed in Supplemental Table S1. Microarray data have been deposited in the Gene Expression Omnibus (GEO) under accession number GSE28629.

\section{GO analyses}

Functional annotation was performed with the Database for Annotation, Visualization, and Integrated Discovery (DAVID; http://david.abcc. ncifcrf.gov). As a cutoff for the functional categories, we chose an FDR (false discovery rate) of 10 .

\section{Teratoma formation assays}

Teratomas were generated by injecting iPSCs and control cells into the flanks of SCID mice. Teratomas were harvested, fixed with $10 \%$ formalin overnight, and sectioned for H\&E staining.

\section{Acknowledgments}

We are grateful to present and past members of the Jaenisch laboratory for their help and critical comments on the manuscript. We particularly thank Dirk Hockemeyer, Frank Soldner, and Caroline Beard. We also acknowledge Emanuela Giacometti, Chris Lengner, Heinz Linhart, Konrad Hochedlinger, Kathrin Plath, Tobias Brambrink, Menno Creyghton, and Grant Welstead for their support. We are grateful to Qing Gao for the histological analysis of teratomas, and Dongdong Fu for processing of histological samples. We would also like to acknowledge George Bell for expert biostatistical analysis, and the Whitehead Genome Technology Core for technical support. M.P. was supported by a predoctoral fellowship from the Ernst Schering Foundation. R.J. was supported by grants R01HD045022 and R01-CA087869 from the NIH.

\section{References}

Blelloch R, Wang Z, Meissner A, Pollard S, Smith A, Jaenisch R. 2006. Reprogramming efficiency following somatic cell nuclear transfer is influenced by the differentiation and methylation state of the donor nucleus. Stem Cells 24: 2007-2013.

Boland MJ, Hazen JL, Nazor KL, Rodriguez AR, Gifford W, Martin G, Kupriyanov S, Baldwin KK. 2009. Adult mice generated from induced pluripotent stem cells. Nature 461: 91-94.

Brambrink T, Foreman R, Welstead GG, Lengner CJ, Wernig M, Suh H, Jaenisch R. 2008. Sequential expression of pluripotency markers during direct reprogramming of mouse somatic cells. Cell Stem Cell 2: 151-159.

Chapman V, Forrester L, Sanford J, Hastie N, Rossant J. 1984. Cell lineage-specific undermethylation of mouse repetitive DNA. Nature 307: 284-286.

Chen T, Ueda Y, Dodge JE, Wang Z, Li E. 2003. Establishment and maintenance of genomic methylation patterns in mouse embryonic stem cells by Dnmt3a and Dnmt3b. Mol Cell Biol 23: 5594-5605.

Deng I, Shoemaker R, Xie B, Gore A, LeProust EM, Antosiewicz-Bourget J, Egli D, Maherali N, Park IH, Yu J, et al. 2009. Targeted bisulfite sequencing reveals changes in DNA methylation associated with nuclear reprogramming. Nat Biotechnol 27: 353-360.

Dodge JE, Okano M, Dick F, Tsujimoto N, Chen T, Wang S, Ueda Y, Dyson N, Li E. 2005. Inactivation of Dnmt3b in mouse embryonic fibroblasts results in DNA hypomethylation, chromosomal instability, and spontaneous immortalization. I Biol Chem 280: 17986-17991.

Doi A, Park IH, Wen B, Murakami P, Aryee MJ, Irizarry R, Herb B, LaddAcosta C, Rho J, Loewer S, et al. 2009. Differential methylation of tissue- and cancer-specific CpG island shores distinguishes human induced pluripotent stem cells, embryonic stem cells, and fibroblasts. Nat Genet 41: 1350-1353.

Feldman N, Gerson A, Fang J, Li E, Zhang Y, Shinkai Y, Cedar H, Bergman Y. 2006. G9a-mediated irreversible epigenetic inactivation of Oct-3/4 during early embryogenesis. Nat Cell Biol 8: 188-194.

Fouse SD, Shen Y, Pellegrini M, Cole S, Meissner A, Van Neste L, Jaenisch R, Fan G. 2008. Promoter CpG methylation contributes to 
ES cell gene regulation in parallel with Oct4/Nanog, PcG complex, and histone H3 K4/K27 trimethylation. Cell Stem Cell 2: 160-169.

Hanna J, Wernig M, Markoulaki S, Sun CW, Meissner A, Cassady JP, Beard C, Brambrink T, Wu LC, Townes TM, et al. 2007. Treatment of sickle cell anemia mouse model with iPS cells generated from autologous skin. Science 318: 1920-1923.

Hanna J, Markoulaki S, Mitalipova M, Cheng AW, Cassady JP, Staerk J, Carey BW, Lengner CJ, Foreman R, Love J, et al. 2009. Metastable pluripotent states in NOD-mouse-derived ESCs. Cell Stem Cell 4: 513-524.

Hochedlinger K, Plath K. 2009. Epigenetic reprogramming and induced pluripotency. Development 136: 509-523.

Hockemeyer D, Soldner F, Cook EG, Gao Q, Mitalipova M, Jaenisch R. 2008. A drug-inducible system for direct reprogramming of human somatic cells to pluripotency. Cell Stem Cell 3: 346-353.

Imamura $M$, Miura $K$, Iwabuchi $K$, Ichisaka $T$, Nakagawa $M$, Lee $J$, Kanatsu-Shinohara M, Shinohara T, Yamanaka S. 2006. Transcriptional repression and DNA hypermethylation of a small set of ES cell marker genes in male germline stem cells. BMC Dev Biol 6: 34 . doi: 10.1186/1471-213X-6-34.

Kaneda M, Okano M, Hata K, Sado T, Tsujimoto N, Li E, Sasaki H. 2004. Essential role for de novo DNA methyltransferase Dnmt3a in paternal and maternal imprinting. Nature 429: 900-903.

Kang L, Wang J, Zhang Y, Kou Z, Gao S. 2009. iPS cells can support fullterm development of tetraploid blastocyst-complemented embryos. Cell Stem Cell 5: 135-138.

Kato Y, Kaneda M, Hata K, Kumaki K, Hisano M, Kohara Y, Okano M, Li E, Nozaki M, Sasaki H. 2007. Role of the Dnmt3 family in de novo methylation of imprinted and repetitive sequences during male germ cell development in the mouse. Hum Mol Genet 16: 22722280.

Li E. 2002. Chromatin modification and epigenetic reprogramming in mammalian development. Nat Rev Genet 3: 662-673.

Lin H, Yamada Y, Nguyen S, Linhart H, Jackson-Grusby L, Meissner A, Meletis K, Lo G, Jaenisch R. 2006. Suppression of intestinal neoplasia by deletion of Dnmt3b. Mol Cell Biol 26: 2976-2983.

Maherali N, Sridharan R, Xie W, Utikal J, Eminli S, Arnold K, Stadtfeld M, Yachechko R, Tchieu J, Jaenisch R, et al. 2007. Directly reprogrammed fibroblasts show global epigenetic remodeling and widespread tissue contribution. Cell Stem Cell 1: 55-70.

Matsui T, Leung D, Miyashita H, Maksakova IA, Miyachi H, Kimura H, Tachibana M, Lorincz MC, Shinkai Y. 2010. Proviral silencing in embryonic stem cells requires the histone methyltransferase ESET. Nature 464: 927-931.

Meissner A, Gnirke A, Bell GW, Ramsahoye B, Lander ES, Jaenisch R. 2005. Reduced representation bisulfite sequencing for comparative high-resolution DNA methylation analysis. Nucleic Acids Res 33: 5868-5877.

Meissner A, Mikkelsen TS, Gu H, Wernig M, Hanna J, Sivachenko A, Zhang X, Bernstein BE, Nusbaum C, Jaffe DB, et al. 2008. Genomescale DNA methylation maps of pluripotent and differentiated cells. Nature 454: 766-770.

Mikkelsen TS, Hanna J, Zhang X, Ku M, Wernig M, Schorderet P, Bernstein BE, Jaenisch R, Lander ES, Meissner A. 2008. Dissecting direct reprogramming through integrative genomic analysis. Nature 454: 49-55.

Nguyen S, Meletis K, Fu D, Jhaveri S, Jaenisch R. 2007. Ablation of de novo DNA methyltransferase Dnmt3a in the nervous system leads to neuromuscular defects and shortened lifespan. Dev Dyn 236: 16631676.

Oda M, Yamagiwa A, Yamamoto S, Nakayama T, Tsumura A, Sasaki $\mathrm{H}_{\text {, }}$ Nakao K, Li E, Okano M. 2006. DNA methylation regulates longrange gene silencing of an X-linked homeobox gene cluster in a lineage-specific manner. Genes \& Dev 20: 3382-3394.

Okano M, Xie S, Li E. 1998. Cloning and characterization of a family of novel mammalian DNA (cytosine-5) methyltransferases. Nat Genet 19: $219-220$

Okano M, Bell DW, Haber DA, Li E. 1999. DNA methyltransferases Dnmt3a and Dnmt3b are essential for de novo methylation and mammalian development. Cell 99: 247-257.

Okita K, Ichisaka T, Yamanaka S. 2007. Generation of germline-competent induced pluripotent stem cells. Nature 448: 313-317.
Sommer CA, Stadtfeld M, Murphy GJ, Hochedlinger K, Kotton DN, Mostoslavsky G. 2009. Induced pluripotent stem cell generation using a single lentiviral stem cell cassette. Stem Cells 27: 543-549.

Stadtfeld M, Maherali N, Breault DT, Hochedlinger K. 2008. Defining molecular cornerstones during fibroblast to iPS cell reprogramming in mouse. Cell Stem Cell 2: 230-240.

Takahashi K, Yamanaka S. 2006. Induction of pluripotent stem cells from mouse embryonic and adult fibroblast cultures by defined factors. Cell 126: 663-676.

Walsh CP, Chaillet JR, Bestor TH. 1998. Transcription of IAP endogenous retroviruses is constrained by cytosine methylation. Nat Genet 20: 116-117.

Wernig M, Meissner A, Foreman R, Brambrink T, Ku M, Hochedlinger K, Bernstein BE, Jaenisch R. 2007. In vitro reprogramming of fibroblasts into a pluripotent ES-cell-like state. Nature 448: 318-324.

Wernig M, Zhao JP, Pruszak J, Hedlund E, Fu D, Soldner F, Broccoli V, Constantine-Paton M, Isacson O, Jaenisch R. 2008. Neurons derived from reprogrammed fibroblasts functionally integrate into the fetal brain and improve symptoms of rats with Parkinson's disease. Proc Natl Acad Sci 105: 5856-5861.

Yamanaka S. 2009. A fresh look at iPS cells. Cell 137: 13-17.

Zhao XY, Li W, Lv Z, Liu L, Tong M, Hai T, Hao J, Guo CL, Ma QW, Wang L, et al. 2009. iPS cells produce viable mice through tetraploid complementation. Nature 461: 86-90.

Zvetkova I, Apedaile A, Ramsahoye B, Mermoud JE, Crompton LA, John R, Feil R, Brockdorff N. 2005. Global hypomethylation of the genome in XX embryonic stem cells. Nat Genet 37: 1274-1279. 


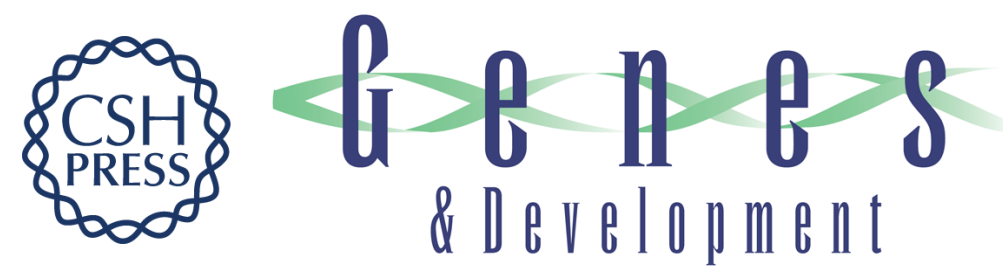

\section{De novo DNA methylation by Dnmt3a and Dnmt3b is dispensable for nuclear reprogramming of somatic cells to a pluripotent state}

Mathias Pawlak and Rudolf Jaenisch

Genes Dev. 2011, 25:

Access the most recent version at doi:10.1101/gad.2039011

Supplemental http://genesdev.cshlp.org/content/suppl/2011/05/10/25.10.1035.DC1
Material

References This article cites 39 articles, 7 of which can be accessed free at:

http://genesdev.cshlp.org/content/25/10/1035.full.html\#ref-list-1

License

Email Alerting

Receive free email alerts when new articles cite this article - sign up in the box at the top

Service

right corner of the article or click here.

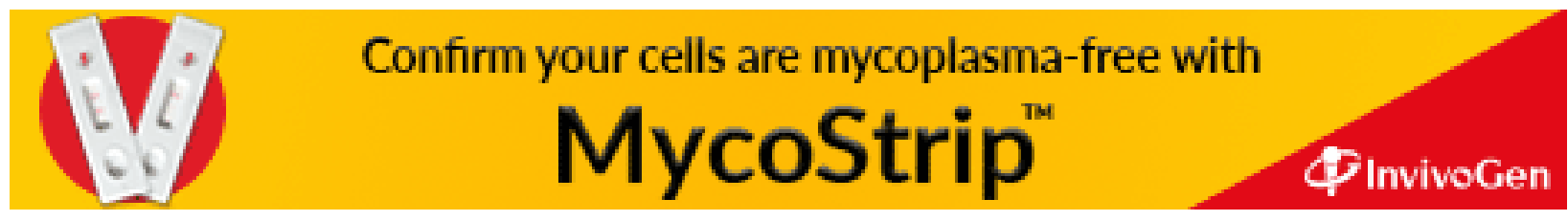

